\title{
Clinical and Pathologic Remission of Pediatric Ulcerative Colitis with Serum-Derived Bovine Immunoglobulin Added to the Standard Treatment Regimen
}

\author{
Rachelle A. Soriano ${ }^{a}$ Asuncion G. Ramos-Soriano ${ }^{b}$ \\ a Division of Global Health, The London School of Economics and Political Science, \\ London, UK; ${ }^{\mathrm{b}}$ Pediatric Gastroenterology and Nutrition, Doctors Hospital of Laredo, \\ Laredo, TX, USA
}

\section{Keywords}

Pediatrics · Refractory ulcerative colitis · Medical food · Oral immunoglobulin

\begin{abstract}
Ulcerative colitis (UC) is a chronic inflammatory bowel disease that is particularly troublesome for pediatric patients, as current therapeutic options consist of biologic agents and steroids which alter the immune response and have the harmful side effect of leaving the patient more susceptible to opportunistic infections and eventual surgery. Another option for therapy exists in the form of serum-derived bovine immunoglobulin/protein isolate (SBI), the key ingredient in a medical food, EnteraGam ${ }^{\circledR}$. The FDA has reviewed the safety of SBI and issued a no challenge letter to the generally recognized as safe (GRAS) findings for this medical food. The product also has no known food or drug interactions, no significant adverse effects, and no contraindications, save for beef allergy. SBI has been shown to induce clinical remission in adult populations and to decrease markers of inflammation in pediatric patients.
\end{abstract}




\section{Case Reports in Gastroenterology}

Soriano and Ramos-Soriano: Remission Achieved in a Refractory Pediatric UC Patient after Addition of SBI

Here, we present a detailed case of pediatric UC, including documentation of mucosal healing and decrease in pediatric UC activity index in a difficult to treat pediatric patient, after the addition of SBI to this patient's treatment regimen.

(C) 2017 The Author(s)

Published by S. Karger AG, Basel

\section{Introduction}

Ulcerative colitis (UC) is a chronic, often lifelong, inflammatory bowel disease (IBD) that affects over a quarter million children in the US [1]. It presents as a remitting and relapsing inflammation of the mucosal lining of varying extent from the rectum to the proximal colon. Affected patients present with crampy abdominal pain, diarrhea, bloody stools, and ultimately growth and nutritional impairment. At least $30 \%$ of pediatric IBD patients also present with extraintestinal manifestations within 15 years of diagnosis, and it has been reported that the incidence of colorectal cancer in 10- to 14-year-old patients with UC is over 118 times greater than that of the control population. The etiopathogenesis of UC is multifactorial with interplay of genetic, immunologic, and environmental factors. Classification of a patient's disease severity using the Pediatric Ulcerative Colitis Activity Index (PUCAI) provides an appropriate guideline in the management of acute disease with use of second-line agents in those with increasing scores [2,3]. The PUCAI defines disease severity with a combination of scores for abdominal pain, rectal bleeding, stool consistency, number of stools in $24 \mathrm{~h}$, nocturnal stools, and activity level to assess disease activity. The score ranges from $0-85$, with $<10$ indicating remission, 10-34 mild disease, 35-64 moderate disease, and 65-85 severe disease (Table 1). Calcineurin inhibitors and biologic agents have been used in severe fulminant cases with the hopes of preventing surgery. However, their use has been associated with toxicities and side effects. Colectomy is warranted in those with life-threatening bleeding and perforation, and is the treatment of choice in patients who are refractory to medical management with long-standing disease and nutritional impairment.

Adjunctive therapies for UC consist of antibiotics and probiotics. Both therapies presumably alter the gut microbiome as a means of controlling disease activity. There are limited studies on antibiotic efficacy in pediatric UC. Probiotics may aid in induction and maintenance of remission in UC and are also believed to decrease the secretion of inflammatory cytokines as well as increase the secretion of anti-inflammatory cytokines, thereby enhancing the mucosal barrier. Several probiotic preparations have been studied in adult and pediatric UC. A medical food probiotic, VSL\#3, appears to hold promise.

Recently, the use of a therapeutic and non-probiotic medical food, serum-derived bovine immunoglobulin/protein isolate (SBI), has been shown to induce clinical and endoscopic remission with a markedly improved Mayo-UC score in an adult with long-standing refractory UC after 2 months of use [4]. Other adult IBD studies support similar results [5, 6]. A recent case report of a 13-year-old pediatric UC patient has also been presented, demonstrating clinical and inflammatory remission based on complete disappearance of symptoms within 4 weeks and decreased fecal calprotectin from $>1,700$ to $<15 \mu \mathrm{g} / \mathrm{g}$ after 3 months of SBI use when added to other standard treatments [7].

SBI in EnteraGam ${ }^{\circledR}$ consists of $>90 \%$ protein, of which nearly $60 \%$ is immunoglobulin ( $>50 \%$ IgG, $\sim 5 \%$ IgM, $\sim 1 \%$ IgA) along with $5 \mathrm{~g}$ of dextrose to aid in dissolution of the protein 


\section{Case Reports in \\ Gastroenterology}

Case Rep Gastroenterol 2017;11:335-343

DOI: 10.1159/000475923

(c) 2017 The Author(s). Published by S. Karger AG, Basel www.karger.com/crg

Soriano and Ramos-Soriano: Remission Achieved in a Refractory Pediatric UC Patient after Addition of SB

in liquids or soft food and trace amounts of the nonallergenic fat, sunflower lecithin, used in the spraying drying process of the product. The overall mechanism of SBI is postulated to involve binding to multiple microbial antigens such as lipopolysaccharide, flagellin, and Clostridium difficile toxins $\mathrm{A}$ and $\mathrm{B}$, aiding in the management of gut barrier function and immune balance by limiting antigen absorption [8,9]. The initial binding event forms antibodyantigen complexes that are effectively too large to translocate through damaged tight junctions within the gut, thus removing a potential chronic trigger of inflammation and leading to an eventual restoration of gut homeostasis [8-10]. Despite its broad activity due to the variety of polyclonal antibodies present, SBI does not adversely affect commensal intestinal bacteria. Previous studies investigating the preparation have demonstrated its ability to reduce signs and symptoms of colitis in both animals and humans [4-7]. Thus, SBI was chosen as an add-on therapy for pediatric UC in this case.

\section{Case Presentation}

We present the case of a 14-year-old, previously well Hispanic-American female teenager. She complained of diffuse abdominal pain for 6 months prior to admission. Associated signs and symptoms consisted of $4.54 \mathrm{~kg}$ of weight loss associated with poor appetite, nausea, and bloody, watery stools with mucus occurring more than 20 times daily. Her past medical history was unremarkable for chronic gastrointestinal disease or prior admission for severe gastrointestinal symptoms. She had a tonsillectomy at the age of 6 years. She had no chronic diseases, no history of frequent antibiotic intake, and no history of travel. Her deceased maternal grandmother had a vague history of colitis. She had lived in a United States border community all her life.

Complete outpatient diagnostic workup by her pediatrician for intestinal parasites and bacterial pathogens was unremarkable. She was initially managed as a case of viral gastroenteritis with conservative measures. She was never febrile. However, the patient's symptoms persisted with multiple school absences and progressive weight loss despite adherence to a bland diet. Her abdominal pain became progressively crampy with increasingly watery, bloody stools with mucus for which she was brought to the emergency room and was subsequently admitted for further evaluation by a pediatric gastroenterologist.

Physical examination upon admission revealed normal temperature, mild tachycardia, and note of generalized pallor. Abdominal examination revealed slight abdominal distension with mild diffuse tenderness and sluggish bowel sounds. Her diagnostic laboratory workup revealed only anemia (hemoglobin $10.5 \mathrm{~g} / \mathrm{dL}$ ) with normal chemistry values. Complete stool studies for viral and bacterial pathogens, parasites, and $C$. difficile toxin were negative. Computerized tomography of the abdomen showed diffuse thickening of the colon. The patient was subsequently evaluated with esophagogastroduodenoscopy and colonoscopy.

Initial biopsies taken in January 2016 when the patient was first diagnosed revealed diffuse active colitis with dense neutrophilic infiltrates producing crypt distortion, cryptitis, and crypt abscesses consistent with a diagnosis of moderate UC which was consistent at the time with a PUCAI score of 60 (Fig. 1). After initial treatment with nightly mesalamine enemas, oral sulfasalazine (500 mg 4 times daily) and short courses of prednisone (40 mg 3 


\section{Case Reports in \\ Gastroenterology}

Case Rep Gastroenterol 2017;11:335-343

(c) 2017 The Author(s). Published by S. Karger AG, Basel www.karger.com/crg

Soriano and Ramos-Soriano: Remission Achieved in a Refractory Pediatric UC Patient after Addition of SBI

times daily for 1 month followed by a taper), there was improvement in the macroscopic findings of a colonoscopy performed in August 2016, but with persistent inflammation (Fig. 2a). The PUCAI score improved from a moderate score of 60 at initial diagnosis to a mild score of 30 in August 2016. Biopsy findings from the August 2016 colonoscopy showed improvement as well, which correlated with a PUCAI score of 30 , but there was still persistent chronic inflammation with active cryptitis and crypt distortion as well as marked depletion of goblet cells (Fig. 3a). The patient responded slowly to the regimen, but with intermittent bouts of abdominal pain and bloody stools. Oral prednisone therapy was restarted in August. Dietary recommendations of low fat and low residue were adhered to during this time. Sulfasalazine was replaced with oral mesalamine $1.2 \mathrm{~g}$ twice daily, but did not appreciably improve symptoms. In November 2016, the patient was started on a nightly course of $5 \mathrm{~g}$ SBI (1 packet EnteraGam ${ }^{\circledR}$ ) added to mesalamine. Crampy abdominal symptoms, blood in the stools, and diarrhea resolved within 2 months of SBI intake.

After the addition of SBI to therapy for 2 months (February 2017), colonoscopy revealed macroscopic normalization of colonic mucosa (Fig. 2b) and biopsy findings demonstrated normal colonic mucosa showing no significant inflammatory activity with restoration of crypt architecture correlating with a PUCAI score of $<10$ (Fig. 3b). The patient had normal stool number and consistency, an absence of bleeding and abdominal pain, an absence of nocturnal stools, and normal activity levels. Laboratory values of complete blood counts revealed normal hemoglobin of $12.6 \mathrm{~g} / \mathrm{dL}$ and normal serum chemistries. The combination of clinical remission of symptoms with normal endoscopic and biopsy findings suggested the patient was in "deep remission."

\section{Discussion}

This case report supports the important role of SBI as an add-on therapy in the management of pediatric UC. The classification of SBI as a medical food lends itself to providing a unique role in the safe management of UC by perhaps decreasing immune activation via binding of microbial components, thereby reducing antigen uptake and release of proinflammatory cytokines and chemokines [8-10]. SBI has been shown to help manage adult IBD [4-6]. Of note, a case of a female adult with refractory UC responded successfully after 2 months of oral steroid therapy and SBI exhibiting endoscopic resolution of disease. Her Mayo-UC score decreased from grade 2 to grade 0 [4]. She was maintained on a low-dose steroid (5 mg per day) and SBI for a year. However, there is limited clinical data to date on the use of SBI in pediatric UC.

There are different outcome measures and definitions of remission in adult and pediatric UC [11]. In adult UC, there are indices to assess disease activity using clinical, endoscopic, quality of life, and histological parameters. The Mayo score is one such index and utilizes both clinical and endoscopic parameters. In pediatrics, Turner et al. [2] suggest that the PUCAI (from NASPGHAN and CDHNF: A Case-Based Monograph on Pediatric IBD [3]) should be used as a primary outcome measure. It is best combined with serological or fecal inflammatory measures of inflammation, imaging, and/or endoscopy. In PUCAI, 6 weighted clinical items (abdominal pain, rectal bleeding, stool consistency, number of stools per $24 \mathrm{~h}$, nocturnal stools, and patient activity level) were used to assess disease severity. Total maximum 


\section{Case Reports in Gastroenterology}

Case Rep Gastroenterol 2017;11:335-343

DOI: $10.1159 / 000475923$

(C) 2017 The Author(s). Published by S. Karger AG, Base www.karger.com/crg

Soriano and Ramos-Soriano: Remission Achieved in a Refractory Pediatric UC Patient after Addition of SB

score was 85 with the following score interpretations: severe $>65$, moderate $35-64$, mild $10-34$, remission $<10$ (Table 1). In clinical practice, colonoscopic findings to confirm remission are not often used because timing of mucosal healing in relation to the duration of subsequent clinical remission varies. However, it has been shown that mucosal healing assessed by macroscopic appearance during endoscopy predicts long-term remission $[12,13]$.

Patient-reported symptoms of clinical remission may predict endoscopic remission, but have yet to be shown to predict the duration of remission. The gold standard for any diagnostic armamentarium in colitis, however, is tissue diagnosis. There is no agreement on the definition of remission in current guidelines, though microscopic or histological healing may be a better predictor than the macroscopic appearance or clinical criteria of time to relapse. Histological assessment with indicators of acute mucosal inflammation such as crypt abscesses, mucin depletion, or an acute inflammatory cell infiltrate were associated with a 2- to 3 -fold increase in the risk of UC relapse during a 12-month follow-up [13]. The presence of dense infiltration of plasma cells in the basal mucosa in patients with quiescent UC has also been associated with a 4.5 -fold increased risk of relapse. Another parameter often studied in the objective measurement of pediatric UC remission is the use of fecal calprotectin. Socalled "deep remission," the combination of clinical remission, normal endoscopic findings, and normal biopsy, is the current goal for all treatments for IBD. Fecal calprotectin in UC has a strong negative predictive value for remission [14]. Recently, a decrease in fecal calprotectin from $>1,700$ to $<15 \mu \mathrm{g} / \mathrm{g}$ and induction of clinical remission after a 3-month addition of SBI in a therapy-refractory UC case where the patient had a limited response to 6-mercaptopurine, mesalamine, and VSL\#3 was described in a teenage UC patient with a 4-year history of relapsing and remitting colitis [7].

Our pediatric case presented with moderate UC and had a 1-year history of relapsing and remitting colitis. Her standard regimen consisted of oral and rectal suspension of mesalamine. She was briefly on corticosteroid therapy for a flare-up of disease activity prior to intake of SBI. She had a healthy diet consisting mainly of white meat and vegetables supplemented by probiotics, which is in line with the dietary recommendations in the specific carbohydrate diet which has been reported to induce remission in pediatric Crohn's disease and UC. In the case presented, photomicrographs of the colonoscopy prior to and after SBI administration validate the assertion with findings of endoscopic remission (Fig. 3a, b). After addition of SBI to therapy, the PUCAI score also decreased to $<10$ within $6-8$ weeks, suggestive of clinical remission. The biopsy findings in this pediatric case after the addition of SBI indicate tissue healing, the third rail in proposed "deep remission" for IBD. It is possible that this patient experienced spontaneous remission, but the temporal nature of symptom relief combined with endoscopy and biopsy findings make this outcome unlikely.

EnteraGam ${ }^{\circledR}$ containing SBI has a potential add-on role to standard treatment protocols in inducing remission in pediatric UC. The pediatric case described here supports the role of immunoglobulins present in SBI that specifically bind to numerous microbe-related inflammatory antigens in targeting one of the multifactorial causes of IBD. With the categorization of EnteraGam ${ }^{\circledR}$ as a medical food, it does not pose any inherent risk for chronic intake as this class of therapeutics requires food-like safety known as generally recognized as safe (GRAS) status. Indeed, the FDA has reviewed the safety of SBI and issued a letter of no challenge to the protein formulation's GRAS status [15]. Further research studies are needed to shed light on SBI's mode of interaction with antibiotics, immunomodulators, anti-inflammatory, and 
biologic agents in the treatment of pediatric IBD. There appears to be promise in the role of long-term bovine serum immunoglobulin as add-on therapy in inducing and sustaining remission in pediatric UC.

\section{Acknowledgements}

We acknowledge Dr. Antonio Alvarez Mendoza, Pathologist, Doctors Hospital of Laredo, for histopathologic diagnosis and providing photomicrographs. We would also like to acknowledge the contributions of Dr. Alex Brewer III and Dr. Bruce P. Burnett for their editorial input and providing background information on SBI.

\section{Statement of Ethics}

This study was conducted in accordance with applicable laws and regulations, including, but not limited to, the International Conference on Harmonisation (ICH), Guideline for Good Clinical Practice (GCP), and the ethical principles that have their origins in the Declaration of Helsinki. The institutional review board (IRB) reviewed and approved the protocol and informed consent form (ICF) before any subjects were enrolled. Before any protocol-required procedures were performed, the subjects signed and dated the IRB-approved ICF.

\section{Disclosure Statement}

Both authors have no conflicts of interest to disclose.

\section{References}

1 Cuffari C: Inflammatory bowel disease in children: a pediatrician's perspective. Minerva Pediatr 2006;58:139-157.

-2 Turner D, Otley AR, Mack D, Hyams J, De Bruijne J, Uusoue K, Walters TD, Zachos M, Mamula P, Beaton DE, Steinhart AH, Griffiths AM: Development, validation, and evaluation of a pediatric ulcerative colitis activity index: a prospective multicenter study. Gastroenterology 2007;133:423-432.

3 Bousvaros A, Turner D, Vitito L, Griefer M, Jenkins J: A Case-Based Monograph Focusing on Pediatric IBD. NASPGHAN and CDHNF, 2009.

4 Beauerle B, Burnett B, Dryden G: Successful management of refractory ulcerative colitis with orally administered serum-derived bovine immunoglobulin therapy. Clin Case Rep Rev 2015;1:90-92. Good L, Panas R: Case series investigating the clinical practice experience of serum-derived bovine immunoglobulin/protein isolate (SBI) in the clinical management of patients with inflammatory bowel disease. J Gastrointest Dig Syst 2015;5:268.

6 Shafran I, Burgunder P, Wei D, Young HE, Klein G, Burnett BP: Management of inflammatory bowel disease with oral serum-derived bovine immunoglobulin. Therap Adv Gastroenterol 2015;8:331-339.

7 Dave M, Burnett BP: Oral serum-derived bovine immunoglobulin therapy to help achieve clinical remission with associated decreases in fecal calprotectin in a pediatric ulcerative colitis patient. Poster presented (\#P62) at NASPGHAN Annual Meeting, Washington, DC, October 7-11, 2015. 


\section{Case Reports in Gastroenterology} \begin{tabular}{l|l}
\hline Case Rep Gastroenterol 2017;11:335-343 \\
\hline DOI: 10.1159/000475923 & $\begin{array}{l}\text { (c) 2017 The Author(s). Published by S. Karger AG, Basel } \\
\text { www.karger.com/crg }\end{array}$
\end{tabular}

Soriano and Ramos-Soriano: Remission Achieved in a Refractory Pediatric UC Patient after Addition of SBI

8 Petschow BW, Burnett B, Shaw AL, Weaver EM, Klein GL: Serum-derived bovine immunoglobulin/protein isolate: postulated mechanism of action for management of enteropathy. Clin Exp Gastroenterol 2014;7:181-190.

9 Horgan A, Maas KJ, Henderson A, Detzel CJ, Weaver EM: Serum-derived bovine immunoglobulin/protein isolate binds to pathogen associated molecular patterns. FASEB J 2014;48:suppl 836.6.

$\checkmark 10$ Detzel CJ, Horgan A, Henderson AL, Petschow BW, Warner CD, Maas KJ, Weaver EM: Bovine Immunoglobulin/protein isolate binds pro-inflammatory bacterial compounds and prevents immune activation in an intestinal co-culture model. PLOS One 2015;10e0120278.

11 Ruemmele FM, Hyams JS, Otley A, Griffiths A, Kolho KL, Dias JA, Levine A, Escher JC, Taminiau J, Veres G, Colombel JF, Vermeire S, Wilson DC, Turner D: Outcome measures for clinical trials in paediatric IBD: an evidence-based, expert-driven practical statement paper of the paediatric ECCO committee. Gut 2015;64:438-446.

12 Rutgeerts P, Vermeire S, Van Assche G: Mucosal healing in inflammatory bowel disease: impossible ideal or therapeutic target? Gut 2007;56:453-455.

-13 Riley SA, Mani V, Goodman MJ, Dutt S, Herd ME: Microscopic activity in ulcerative colitis: what does it mean? Gut 1991;32:174-178.

14 Xiang JY, Ouyang Q, Li GD, Xiao NP: Clinical value of fecal calprotectin in determining disease activity of ulcerative colitis. World J Gastroenterol 2008;14:53-57. http://www.accessdata.fda.gov/scripts/fdcc/?set=GRASNotices\&id=255 (accessed April 2017).
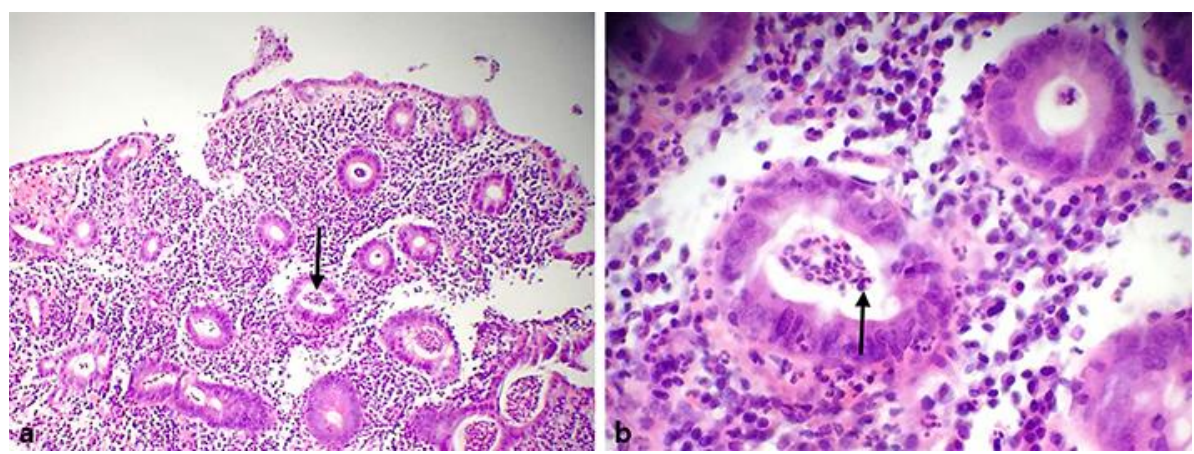

Fig. 1. a Low-power view $(10 \times)$ of January 2016 colonic mucosal biopsy of the patient showing diffuse active colitis with dense neutrophilic infiltrates producing crypt distortion, cryptitis, and crypt abscesses (arrow) consistent with the diagnosis of ulcerative colitis. b High-power view $(40 \times)$ of the same colonic biopsy (January 2016) showing dense chronic inflammatory infiltrates of lymphocytes, plasma cells, and eosinophils (arrow). (Soriano and Soriano; histopathology by Antonio Alvarez Mendoza, MD.) 


\section{Case Reports in Gastroenterology}

\begin{tabular}{l|l}
\hline Case Rep Gastroenterol 2017;11:335-343 \\
\hline DOI: 10.1159/000475923 & $\begin{array}{l}\text { (c) } 2017 \text { The Author(s). Published by S. Karger AG, Basel } \\
\text { www.karger.com/crg }\end{array}$ \\
\hline
\end{tabular}

Soriano and Ramos-Soriano: Remission Achieved in a Refractory Pediatric UC Patient after Addition of SBI
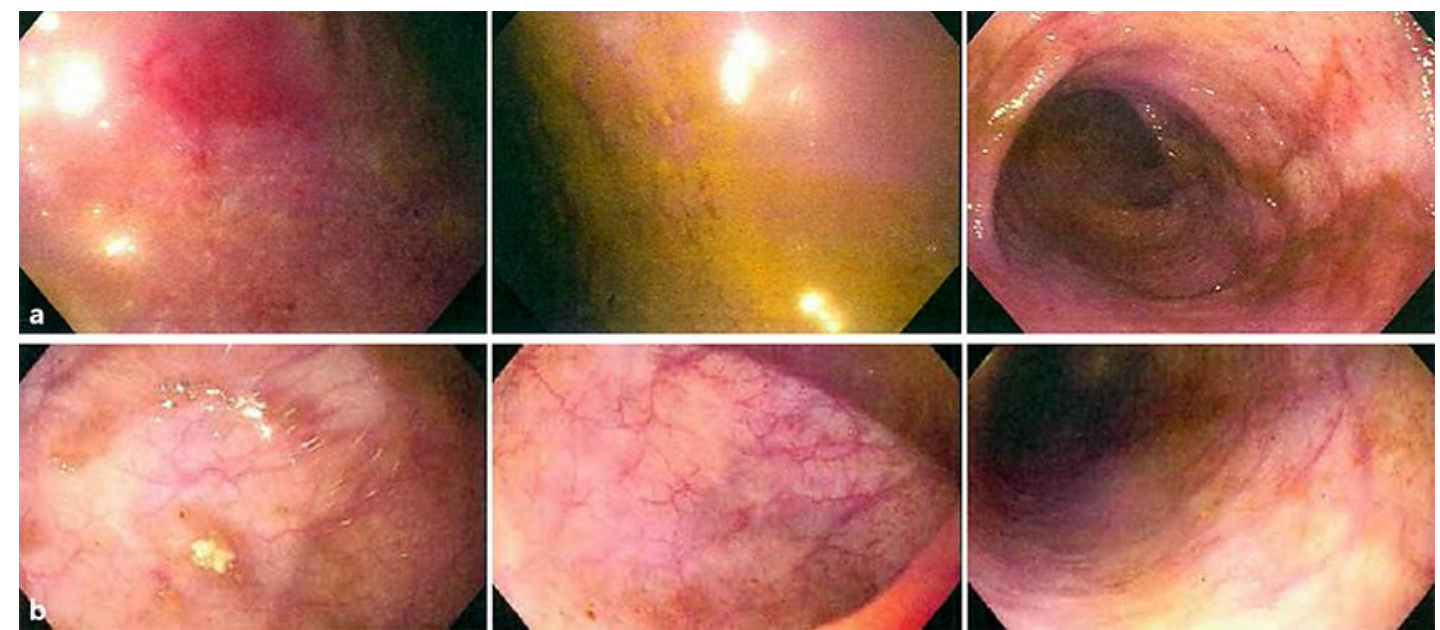

Fig. 2. Colonoscopy performed in August 2016 prior to SBI administration (a) compared to colonoscopy in February 2017 after 2 months of SBI administration (b).
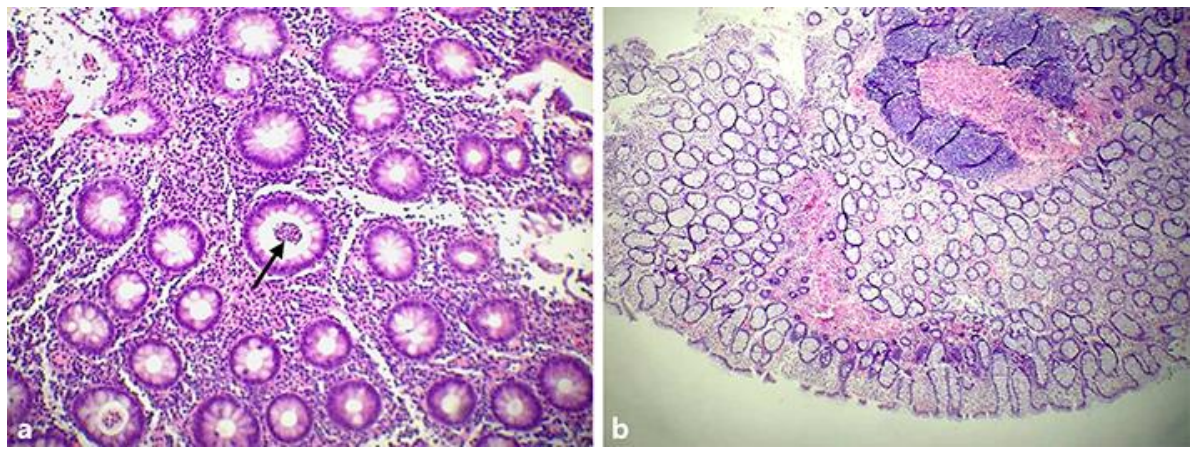

Fig. 3. a Low-power (10×) biopsy view of colonic mucosa (August 2016 image) showing persistent chronic inflammation with active cryptitis, crypt distortion, and marked depletion of goblet cells. Note the persistence of crypt abscess and surface ulcerations (arrow). b February 2017 lower-power (4×) image showing no significant inflammatory activity with restoration of crypt architecture. (Soriano and Soriano; histopathology by Antonio Alvarez Mendoza, MD.) 
Soriano and Ramos-Soriano: Remission Achieved in a Refractory Pediatric UC Patient after Addition of SBI

Table 1. Pediatric Ulcerative Colitis Activity Index (PUCAI)

\begin{tabular}{ll}
\hline Item & Category/points \\
\hline Abdominal pain & No pain $=0$ \\
& Pain can be ignored $=5$ \\
& Pain cannot be ignored $=10$ \\
\hline Rectal bleeding & None $=0$ \\
& Small amount only, in less than $50 \%$ of stools $=20$ \\
& Small amount with most stools $=20$ \\
& Largest amount $(50 \%$ of the stool content) $=30$ \\
\hline Stool consistency of most stools & Formed $=0$ \\
& Partially formed $=5$ \\
& Completely unformed $=10$ \\
\hline Number of stools per $24 \mathrm{~h}$ & $0-2=0$ \\
& $3-5=5$ \\
& $6-8=10$ \\
& $>8=15$ \\
\hline Nocturnal stools (any episode causing & No $=0$ \\
wakening) & Yes $=10$ \\
\hline Activity level & No limitation of activity $=0$ \\
& Occasional limitation of activity $=5$ \\
& Severe restricted activity $=10$ \\
\hline & Sum of PUCAI ( $0-85)$ \\
\hline & \\
& \\
&
\end{tabular}

Disease severity is defined by the following scores: severe, $\geq 65$; moderate, 35-64; mild, 10-34; remission (inactive disease), $<10$. 